\title{
HbA1c as a diagnostic tool in diabetes mellitus
}

\author{
Jens P. Berg \\ Department of Medical Biochemistry, Institute of Clinical Medicine, University of Oslo and \\ Oslo University Hospital, Oslo, Norway \\ Correspondence: Jens P. Berg, Department of Medical Biochemistry, Oslo University Hospital, Postboks 4950 Nydalen, NO-0424 Oslo, Norway \\ E-mail: j.p.berg@medisin.uio.no Telephone: +4723027251Ｍobile: +47 48496950
}

\begin{abstract}
SUMMARY
Hemoglobin A1c (HbA1c) expresses the ratio between glycated HbA1 and total HbA1 and reflects the average plasma glucose concentration the preceding 2-3 months. It has become the most important marker of chronic hyperglycemia and is closely correlated to late complications of diabetes. The analysis has now been standardized and traceable to an international reference material and reference methods. Standardization of the HbA1c analyses paved the way for it to become the primary tool for diagnosing diabetes. Despite some limitations in which $\mathrm{HbA} 1 \mathrm{c}$ is not representative of average glucose concentration, the inclusion of HbA1c as a diagnostic tool will simplify the diagnostics of diabetes compared with the glucose criteria, and hopefully improve the prevention of late complications of diabetes.
\end{abstract}

\section{CHANGING DIAGNOSTIC CRITERIA OF DIABETES}

Chronic hyperglycemia is the hallmark of diabetes mellitus. The diagnosis has until recently been based upon the detection of elevated plasma glucose levels either fasting or two hours after an oral glucose (75 g) tolerance test (OGTT) (1). The aim was initially to identify persons at high risk of developing acute symptoms and signs of hyperglycemia. In 1997 the American Diabetes Association recommended that the diagnostic levels of glucose should be based on the risk of developing late complications of chronic hyperglycemia instead of acute symptoms of hyperglycemia (2). The cut-off levels were determined by epidemiologic studies showing an association between glucose concentrations and retinopathy, which is regarded as the most diabetes specific late complication. The risk of developing retinopathy is characterized by relatively well defined threshold levels of fasting and OGTT glucose concentrations. Consequently, the diagnostic level of fasting plasma glucose was lowered from $\geq 7.8$ to $\geq 7.0 \mathrm{mmol} / \mathrm{L}$, whereas the OGTT level at $\geq 11.1 \mathrm{mmol} / \mathrm{L}$ remained unchanged. In contrast, the risk of developing chronic hyperglycemia and glucose values above the diagnostic criteria for diabetes shows no threshold, but increases along a continuum of increasing plasma glucose concentrations below the diagnostic levels.

Hemoglobin Alc $(\mathrm{HbA} 1 \mathrm{c})$ is a hemoglobin variant that is formed when glucose binds covalently to the beta-chain of HbA (for references see (3)). The initial Schiff base which is formed is subsequently rearranged to a stable Amadori product. The level of HbA1c closely reflects the average plasma glucose concentration the preceding 2-3 months (4). Approximately 50\% of the amount of $\mathrm{HbAlc}$ is determined by the average glucose the last preceding month. The association between $\mathrm{HbA} 1 \mathrm{c}$ and both glucose concentration and diabetic late complications has been extensively docu- mented, early by a Norwegian study (5), and later in particular by the DCCT and UKPDS studies of type 1 and type 2 diabetes, respectively $(6,7)$. HbA1c is our most important biomarker for glycemia control in diabetes. Although measurement of HbA1c has become essential for the follow-up of patients with diabetes, diagnostic use of $\mathrm{HbAlc}$ has been rejected at several revisions of the diagnostic criteria mainly with referral to the lack of standardization of the analysis. However, the treatment goals for diabetes have been defined in HbA1c units calibrated to the DCCT study. The need for a uniform definition of reference material and reference measurement systems was imminent when the International Federation of Clinical Chemistry (IFCC) defined that $\mathrm{HbA} 1 \mathrm{c}$ is the valine glycated N-terminal hexapeptide of the beta-chain of hemoglobin A1 (8). $\mathrm{HbA} 1 \mathrm{c}$ is the concentration ratio between the glycated and the total glycated and non-glycated hexapeptide. In addition two reference methods for $\mathrm{HbA} 1 \mathrm{c}$ measurements were defined and described. All vendors of $\mathrm{HbA1c}$ instruments in Norway have to certify that the results are traceable to the IFCC reference material and method.

Standardization of the HbAlc analysis for the follow-up of patients with diabetes, paved the way for its diagnostic use. Systematic differences between instruments became smaller, and total allowable error in external quality controls became tighter. Total error shrank in the US National Glycohemoglobin Standardization Program (NGSP) from $15 \%$ in 2007 to $7 \%$ in 2011, which corresponds to the quality of glucose measurements (9). An international Expert Committee revived in 2009 the possibility of diagnostic use of HbA1c in the wake of its standardization (10). The diagnostic cut-off was suggested at $\geq 6.5 \%$ (48 $\mathrm{mmol} / \mathrm{mol}$ ) which corresponds to an increased risk of nonproliferative diabetic retinopathy. A report from a World Health Organization (WHO) consultation accepted the diagnostic use of $\mathrm{HbAlc}$ at $\geq 6.5 \%$ in 
Table 1. Diagnostic criteria for diabetes in Norway ${ }^{1}$

\begin{tabular}{|l|}
\hline 1. HbAlc $\geq 6.5 \%{ }^{2}$ \\
OR: If $\mathrm{HbA} 1 \mathrm{c}$ is regarded inadequate \\
2. Fasting plasma glucose $\geq 7.0 \mathrm{mmol} / \mathrm{L}$ \\
\hline OR: \\
3. 2-h plasma glucose $\geq 11.1 \mathrm{mmol} / \mathrm{L}$ after an oral glucose tolerance test \\
OR: \\
4. Random plasma glucose $\geq 11.1 \mathrm{mmol} / \mathrm{L}$ in a patient with classic symptoms of hyperglycemia or hyperglycemic crisis. \\
\hline
\end{tabular}

${ }^{1}$ Criteria 1-3 have to be confirmed by repeat testing.

${ }^{2}$ Analyzed by a laboratory fulfilling the diagnostic quality criteria.

2011 (11), but did not define a subgroup of high-risk individuals based on HbAlc levels such as suggested by the International Expert Committee (HbA1c 6.06.4\%) (10) and the American Diabetes Association (HbA1c 5.7-6.4\%) (1). The Norwegian Directorate of Health endorsed the WHO report in September 2012, and modified it so that HbAlc is the recommended diagnostic analysis for diabetes. Glucose measurements (fasting or OGTT) should be used when HbA1c is unreliable as a measure of the level of glycemia. The current diagnostic criteria for diabetes in Norway are listed in Table 1.

\section{DiAgNOSTIC CONSIDERATIONS}

For correct diagnostic use of HbA1c it is essential that the result is 1) representative of the patient's glycemia, 2) within acceptable limits of deviation from the "true value", and 3 ) correctly interpreted according to the diagnostic criteria.

Although there is a strong and positive correlation between average plasma glucose concentration and $\mathrm{HbA1c}$ levels, large individual differences exist (for references see $(1,10,12,13)$. A rapid and stable change in plasma glucose level requires up to 30 days for a new steady state level of HbAlc (14). HbAlc is unsuitable to diagnose rapidly progressing type 1 diabetes. Conditions affecting the glycation rate of hemoglobin differ between individuals. The life span of erythrocytes markedly influences HbAlc levels within each red blood cell and the average HbA1c level of the individual. HbA1c levels higher than expected based on average glucose concentrations are expected when the average erythrocyte age is increased. This can be seen either caused by reduced erythrocyte synthesis such as iron deficiency anemia, vitamin B12 deficiency, chronic kidney disease, and bone marrow suppression or by reduced hemolysis after splenectomy. A lower HbA1c than expected is observed in conditions with increased red blood cell synthesis such as recent iron substitution in iron deficiency anemia or treatment with erythropoietin. Increased hemolysis will reduce the average erythrocyte age and subsequently result in lower HbAlc levels than expected. Among the ten genetic loci associated with $\mathrm{HbA}$ 1c level in Caucasians, three are in loci associated with fasting glucose levels, whereas seven are in genes related to erythrocyte physiology (15). A decision to use $\mathrm{HbA1c}$ diagnostically must also consider the probability that the patient has hemoglobin variants that may interfere with the analysis. A list of variants and their influence on $\mathrm{HbAlc}$ measurements is presented by the NGSP website (http://www.ngsp.org/interf.asp). Gestational diabetes has its own glucose based diagnostic criteria and has not included $\mathrm{HbA} 1 \mathrm{c}$.

HbA1c reference values of native samples are determined by certified laboratories. The WHO report did not specify quality criteria for diagnostic use of $\mathrm{HbA1c}$, but stated that the analysis must be traceable to the IFCC standardization and that the laboratory has to participate in external quality assessment programs (16). In Norway all instruments are calibrated according to the IFCC reference material. The results are converted from IFCC format ( $\mathrm{mmol} / \mathrm{mol})$ to the NGSP level (\%), which is similar to DCCT level, by the formula (8):

$$
\text { NGSP }(\%)=0.0915 * \operatorname{IFCC}(\mathrm{mmol} / \mathrm{mol})+2.15
$$

The external quality assessment program in Norway is organized by NOKLUS both for laboratories in hospitals and in primary care. The native control sample which is distributed to the laboratories is given a reference value for $\mathrm{HbA} 1 \mathrm{c}$ by a collaborating reference laboratory. The total allowable error at the diagnostic level of $6.5 \%$ is set to $7 \%$. This means that for a reported $\mathrm{HbAlc}$ value to be acceptable it has to be between $6.0-7.0 \%$ when the reference value of the sample is $6.5 \%$. A total allowable error of $7 \%$ is in accordance with the NGSP requirements. In addition the laboratory has to document an internal quality control coefficient of variation $(\mathrm{CV}) \leq 2 \%$. The external quality requirement will be fulfilled by more than $90 \%$ of the participating Norwegian laboratories (personal communication). The limit of the internal quality control $\mathrm{CV}$ is quite strict, but is based on reported desirable quality performance of $\mathrm{HbAlc}$ (9). It remains to be seen how these requirements will influence the laboratories willingness and efforts to obtain diagnostic HbA1c quality.

Diabetes can be diagnosed if HbA1c, fasting plasma glucose or the OGTT result is above the cut-off level in two independent tests (1). HbAlc is the preferred 


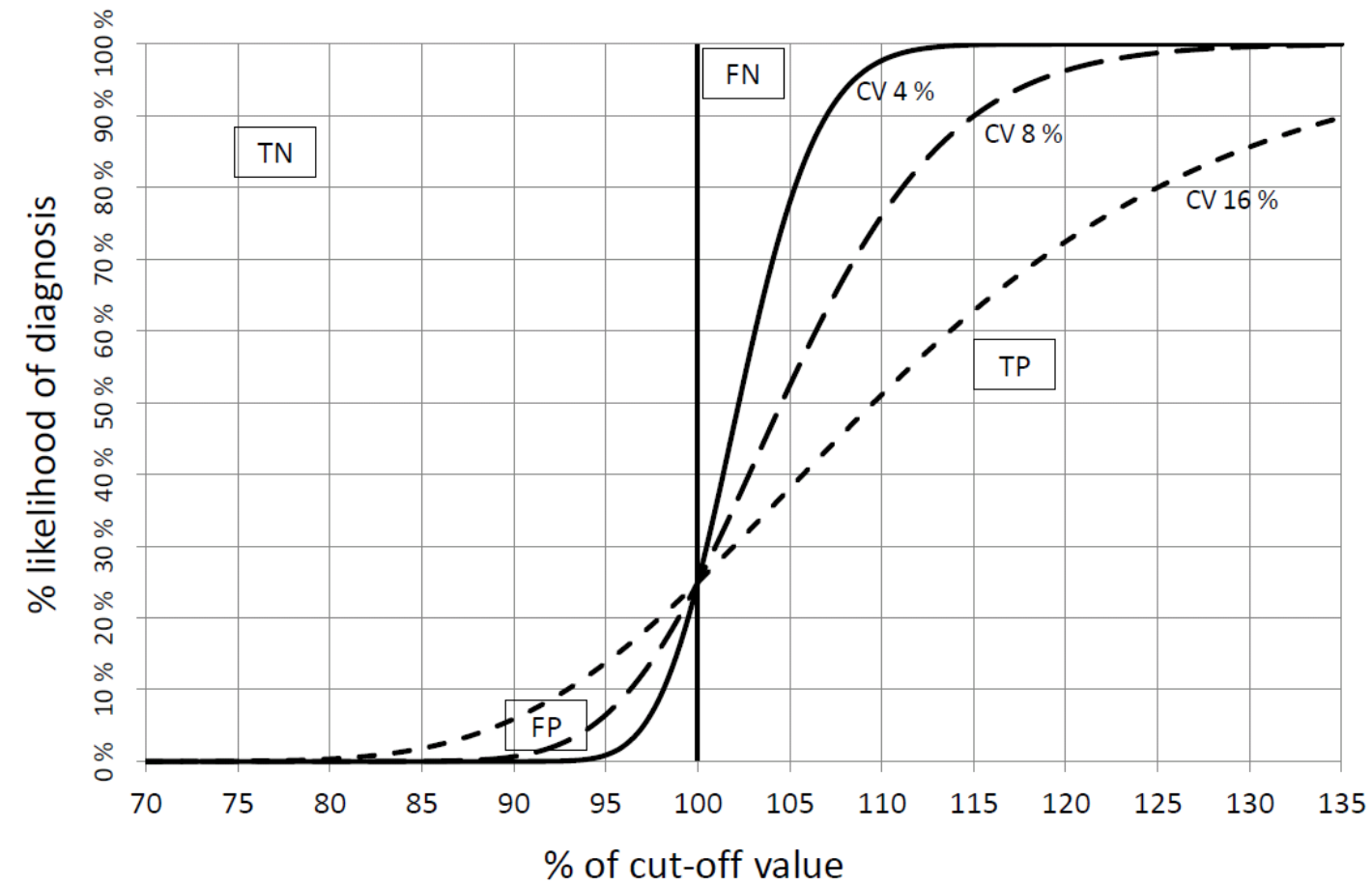

Figure 1. Per cent likelihood of diabetes diagnosis in individuals with a true value for the diagnostic test expressed as per cent of the diagnostic-cut-off value at different coefficients of variation (CVs). The likelihood is estimated based on two consecutive tests equal to or above the cut-off level of the same test in the same individual using CVs of $4 \%, 8 \%$, and $16 \%$ representative of $\mathrm{HbA1c}$, fasting plasma glucose, and 2-h glucose after OGTT, respectively. The model assumes that the tests are performed without bias. $\mathrm{TN}=$ true negatives, $\mathrm{FP}=$ false positives, $\mathrm{FN}=$ false negatives, and $\mathrm{TP}=$ true positives.

test. An elevated test result has to be confirmed by a second test of the same substance above the cut-off level normally a few weeks later, but the time between the two tests has not been specified. If for example both $\mathrm{HbAlc}$ and fasting plasma glucose are above the cut-off level, the patient has diabetes because two independent tests are elevated. If fasting plasma glucose is above the cut-off, and $\mathrm{HbAlc}$ below, fasting plasma glucose should be controlled and confirmed elevated before a diagnosis can be established. If the patient has symptoms or signs of diabetes, one test result above the cut-off is sufficient.

It has been argued that the implementation of $\mathrm{HbAlc}$ among the diagnostic tests will disturb the epidemiologic data of diabetes. When screening a population without diabetes with $\mathrm{HbAlc}$, fasting plasma glucose and OGTT, it is generally observed that the fraction with OGTT results above the diagnostic threshold will be largest, elevated fasting plasma glucose comes second, whereas the fraction with HbAlc above the cut-off is the smallest (17). It has been claimed that HbA1c will detect fewer patients with diabetes than the glucose criteria. This is based on the assumption that an elevated result will be equally reproduced at the control test, which is required for the diagnosis, regardless of method employed. However, the total coefficient of variation (CV) including biological and analytical variation within individuals is much higher for OGTT compared with fasting plasma glucose which is intermediate and $\mathrm{HbAlc}$ with the lowest $\mathrm{CV}$ of approximately $4 \%(18-20)$. An elevated HbAlc level is more likely to be reproduced and confirm a diagnosis of diabetes compared with an elevated OGTT result. When the true value of an individual is $100 \%$ of the threshold value, the likelihood of having two consecutive tests equal to or above the cut-off is $25 \%$ independent of the CVs when systematic bias is excluded (Figure 1). The likelihood increases to almost $100 \%$ when the true value is $110 \%$ of the cut-off value for a test with a total $\mathrm{CV}$ of $4 \%$, which is typical performance of HbAlc. This corresponds to a HbAlc level of $7.2 \%$. For fasting plasma glucose the $\mathrm{CV}$ is typically at $8 \%$. A true glucose level $10 \%$ higher than the cut-off $(7.7 \mathrm{mmol} / \mathrm{L})$ results in a likelihood of diagnosing diabetes at approximately $75 \%$. The likelihood is reduced to $50 \%$ when the individual true concentration of plasma glucose after OGTT (typical CV 16\%) is $110 \%$ of the cut-off $(12.2 \mathrm{mmol} / \mathrm{L})$. True fasting plasma glucose level has to be at least $8.7 \mathrm{mmol} / \mathrm{L}$ in order to obtain a similar level of diabetes diagnostic likelihood as a true HbAlc value of $7.2 \%$ due to the differences in CVs.

\section{CONCLUSION}

International standardization of the $\mathrm{HbA} 1 \mathrm{c}$ analysis has made it suitable not only for the follow-up of patients with diabetes, but also for diagnostic purpose. 
Although the interpretation of HbA1c as a biomarker of average plasma glucose has some limitations in particular related to erythrocyte turnover and metabolism, $\mathrm{HbA} 1 \mathrm{c}$ has several advantages and requires no fasting or time-consuming testing and is stable and more conveniently handled than plasma glucose. Using HbA1c as the primary diagnostic criterion should make it easier to detect diabetes at an early stage. Early identi- fication of patients with diabetes should be encouraged as it is fundamental for successful prevention of late complications of diabetes.

\section{ACKNOWLEDGEMENT}

The discussion and estimation of diagnostic likelihood in collaboration with Daniel Sachse is greatly appreciated.

\section{REFERENCES}

1. American Diabetes Association. Diagnosis and classification of diabetes mellitus. Diabetes Care 2010; 33 (Suppl 1): S62-S69.

2. The Expert Committee on the Diagnosis and Classification of Diabetes Mellitus. Report of the Expert Committee on the Diagnosis and Classification of Diabetes Mellitus. Diabetes Care 1997; 20 (7): 1183-97.

3. Weykamp C, John W, Mosca A. A review of the challenge in measuring hemoglobin A1c. J Diabetes Sci Technol 2009; 3 (3): 439-45.

4. Nathan DM, Kuenen J, Borg R, Zheng H, Schoenfeld D, Heine RJ. Translating the A1C assay into estimated average glucose values. Diabetes Care 2008; 31 (8): 1473-8.

5. Dahl-Jørgensen K, Brinchmann-Hansen O, Hanssen KF, Ganes T, Kierulf P, Smeland E, et al. Effect of near normoglycaemia for two years on progression of early diabetic retinopathy, nephropathy, and neuropathy: the Oslo study. BMJ 1986; 293 (6556): 1195-9.

6. The Diabetes Control and Complications Trial Research Group. The effect of intensive treatment of diabetes on the development and progression of long-term complications in insulin-dependent diabetes mellitus. $N$ Engl J Med 1993; 329 (14): 977-86.

7. UK Prospective Diabetes Study (UKPDS) Group. Intensive blood-glucose control with sulphonylureas or insulin compared with conventional treatment and risk of complications in patients with type 2 diabetes (UKPDS 33). Lancet 1998; 352 (9131): 837-53.

8. Hoelzel W, Weykamp C, Jeppsson JO, Miedema K, Barr JR, Goodall I, et al. IFCC reference system for measurement of hemoglobin A1c in human blood and the national standardization schemes in the United States, Japan, and Sweden: a method-comparison study. Clin Chem 2004; 50 (1): 166-74.

9. Little RR, Rohlfing CL, Sacks DB. Status of hemoglobin A1c measurement and goals for improvement: From chaos to order for improving diabetes care. Clin Chem 2011; 57 (2): 205-14.

10. International Expert Committee report on the role of the $\mathrm{A} 1 \mathrm{C}$ assay in the diagnosis of diabetes. Diabetes Care 2009; 32 (7): 1327-34.

11. Use of glycated hemoglobin (HbA1c) in the diagnosis of diabetes mellitus. Abbreviated report of a WHO consultation. Geneva: World Health Organization, 2011.

12. Hare MJL, Shaw JE, Zimmet PZ. Current controversies in the use of haemoglobin A1c. J Intern Med 2012; 271 (3): 227-36.

13. Malkani S, Mordes JP. Implications of using hemoglobin A1C for diagnosing diabetes mellitus. Am J Med 2011; 124 (5): 395-401.

14. Mortensen HB, Vølund A. Application of a biokinetic model for prediction and assessment of glycated haemoglobins in diabetic patients. Scand J Clin Lab Invest 1988; 48 (6): 595-602.

15. Soranzo N, Sanna S, Wheeler E, Gieger C, Radke D, Dupuis J, et al. Common variants at 10 genomic loci influence hemoglobin $A_{1 C}$ levels via glycemic and nonglycemic pathways. Diabetes 2010; 59 (12): 3229-39.

16. Definition and diagnosis of diabetes mellitus and intermediate hyperglycaemia. Report of WHO/IDF consultation. Geneva: World Health Organization, International Diabetes Federation, 2006.

17. Cowie CC, Rust KF, Byrd-Holt DD, Gregg EW, Ford ES, Geiss LS, et al. Prevalence of diabetes and high risk for diabetes using A1C criteria in the U.S. population in 1988-2006. Diabetes Care 2010; 33 (3): 562-8.

18. Rasmussen SS, Glümer C, Sandbaek A, Lauritzen T, Carstensen B, Borch-Johnsen K. Short-term reproducibility of impaired fasting glycaemia, impaired glucose tolerance and diabetes: The ADDITION study, DK. Diabetes Res Clin Pract 2008; 80 (1): 146-52.

19. Utzschneider KM, Prigeon RL, Tong J, Gerchman F, Carr DB, Zraika S, et al. Within-subject variability of measures of beta cell function derived from a 2-h OGTT: implications for research studies. Diabetologia 2007; 50 (12): 2516-25.

20. Selvin E, Crainiceanu CM, Brancati FL, Coresh J. Short-term variability in measures of glycemia and implications for the classification of diabetes. Arch Intern Med 2007; 167 (14): 1545-51. 